\title{
Experimental Studies of Helical Nonneutral Plasmas with a Helical Magnetic Axis
}

\author{
Daichi SUGIMOTO, Haruhiko HIMURA, Hiroyuki OKADA ${ }^{1)}$, \\ Satoshi YAMAMOTO ${ }^{1)}$, Shinji KOBAYASHI ${ }^{1)}$, Tohru MIZUUCHI ${ }^{1)}$, \\ Sadao MASAMUNE and Fumimichi SANO ${ }^{1)}$ \\ Department of Electronics, Kyoto Institute of Technology, Matsugasaki, Kyoto 606-0855, Japan \\ ${ }^{1)}$ Institute of Advanced Energy, Kyoto University, Gokasho, Uji, Kyoto 611-0011, Japan
}

(Received 8 December 2009 / Accepted 7 April 2010)

\begin{abstract}
Toroidal nonneutral plasmas confined on helical magnetic surfaces with a helical axis are experimentally studied for the first time. Variations in space potential $\varphi_{\mathrm{s}}$ and electron density $n_{\mathrm{e}}$ on closed helical magnetic surfaces are clearly observed similar to those of helical plasmas with plane axes in past experiments. The measured data in this experiment are compared with numerical results obtained from Poisson's equation applied to poloidal cross sections of Heliotron J.
\end{abstract}

(C) 2010 The Japan Society of Plasma Science and Nuclear Fusion Research

Keywords: toroidal nonneutral plasma, pure electron plasma, magnetic surface confinement, variation of plasma parameters on magnetic surface

DOI: $10.1585 /$ pfr.5.S2028

\section{Introduction}

Experiments on nonneutral plasmas have been conducted on various machines such as the Penning trap [1], the Paul trap [2], and toroidal devices [3]. Recently, toroidal nonneutral plasmas confined on helical magnetic surfaces (HMSes) have been conducted $[4,5]$. Unique phenomena of nonneutral helical plasmas have been observed, that have never been seen in neutral helical plasmas. For example, experiments on the Compact Helical System (CHS) have clearly revealed that injected electrons can penetrate quickly into the HMS from outside the last closed flux surface (LCFS) [4]. To explain this phenomenon, numerical work has been performed, and recently, outward orbits that extend to the inward part of the closed HMS have been found [6]. Another significant feature is that the plasma equipotential surfaces do not coincide with the HMS [7]. Thus, the space potential $\varphi_{\mathrm{s}}$ is not constant along magnetic field lines. This was also observed in experiments where data were measured along the helical plane axis of the Columbia Non-neutral Torus (CNT) device [8]. In addition, the CNT group had compared the obtained data with the result output from the magnetohydrodynamic (MHD) equilibrium code they developed [8,9]. However, all of those studies were performed only in devices with plane axes. No experiment has been conducted yet with a machine having a helical magnetic axis . To systematically investigate basic plasma physics in toroidal nonneutral plasmas, experimental studies in a device having a helical magnetic axis are thus necessary.

The Heliotron J machine [10] at Kyoto University is a

author'se-mail: himura@kit.ac.jp medium-sized helical machine having a helical magnetic axis. Unlike the CHS device, it forms quasi-poloidally symmetrical magnetic surfaces, and the magnetic field strength $B$ is almost uniform on poloidal cross sections of straight-stretch portions of the HMS in the device. This property may produce quasi-isodynamic plasmas with a confinement time longer than that of past CHS nonneutral plasmas. Also, like the CHS, Heliotron $\mathrm{J}$ can produce a static closed HMS, and the LCFS is completely detached from the vacuum chamber. These properties enable a systematic study of helical nonneutral plasmas. For the reasons mentioned above, we have initiated experiments on helical nonneutral plasmas in Heliotron J.

In this paper, we report the first results of experiments on nonneutral plasmas confined on the HMS of Heliotron $J$. Even in this machine, variations in $\varphi_{\mathrm{s}}$ and the electron density $n_{\mathrm{e}}$ on the closed HMS are clearly observed. Those measured data seem to qualitatively agree with results obtained from the one-dimensional Poisson's equation applied to poloidal cross sections of Heliotron J. In section 2, the experimental setup is explained. Experimental data are presented in section 3 . These data are compared with the one-dimensional simulation in section 4. Finally, in section 5 , a summary is given.

\section{Experimental Setup}

Experiments are conducted on Heliotron $\mathrm{J}$ whitch has a helical magnetic axis. The average major and minor radii of the machine are 1.2 and $0.38 \mathrm{~m}$, respectively. Figure 1 shows a schematic drawing of a top view of Heliotron J. Four poloidal cross sections where probes are installed 


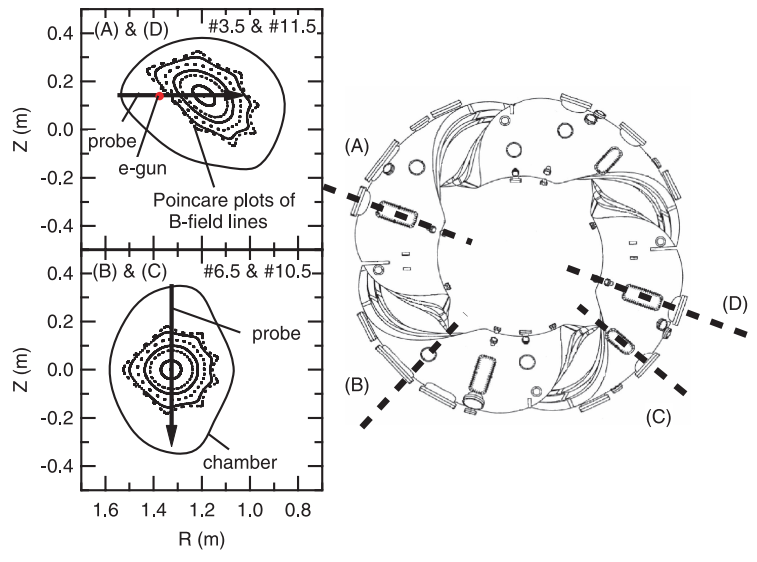

Fig. 1 Top view of Heliotron $\mathrm{J}$ and Poincare maps of poloidal cross sections of probe insertion locations. Arrows indicate the positions and directions of the probes. Electrons are injected outside the LCFS at the 3.5 poloidal cross section [red dot in (A), top left panel].

during experiments are also shown. The toroidal angles $\phi$ of the four poloidal cross sections are $71.05^{\circ}, 138.55^{\circ}$, $228.55^{\circ}$ and $251.05^{\circ}$, respectively. They are labeled (A), (B), (C), and (D) in Fig. 1. Henceforth, we call those positions as the $3.5,6.5,10.5$, and 11.5 poloidal cross sections, respectively. Since Heliotron $\mathrm{J}$ has $L=1$ and $M=4$, the same HMS appears at each rotated $90^{\circ}$ in the toroidal direction.

Electrons are launched from the electron gun (e-gun) [11], whitch has $\mathrm{a} \mathrm{LaB}_{6}$ emitter as the cathode. The $\mathrm{LaB}_{6}$ cathode is quadrate $(1.5 \mathrm{~cm}$ each), and the emitter also has a quadrille shape with tungsten wires. The e-gun is inserted horizontally along the $r$ axis and set outside the LCFS on the 3.5 poloidal cross section. The acceleration voltage $V_{\text {acc }}$ of the electrons is variable, but it is fixed at $V_{\text {acc }}=-600 \mathrm{~V}$ in this experiment. The pulse width of $V_{\text {acc }}$ applied to the cathode is also variable in the range between $\sim 10 \mu \mathrm{s}$ and $\sim 100 \mathrm{~ms}$, but it is likewise fixed at $10 \mathrm{~ms}$ for this research. The electron temperature $T_{\mathrm{e}}, \varphi_{\mathrm{s}}$, and $n_{\mathrm{e}}$ are measured with the same probing method employed in past CHS experiments [7]. The probe can be used as not only an emissive probe but also a Langmuir probe. In Heliotron $\mathrm{J}$ experiments, the probe is installed on the $6.5,10.5$, and 11.5 poloidal cross sections. The probe shaft is sufficiently long to reach the inboard edge of the LCFS.

The magnetic field $B$ of Heliotron $\mathrm{J}$ is static in this study. The strength of $B$ on the helical magnetic axis of the HMS is about $0.3 \mathrm{kG}$. A typical vacuum pressure is about $2 \times 10^{-8}$ Torr.

\section{Experiments}

\subsection{Measured $I_{\mathrm{p}}-V$ characteristic curve}

Figure 2 shows a typical current-voltage $\left(I_{\mathrm{p}}-V\right)$ characteristic curve of the probe described above. No inflection point near $\varphi_{\mathrm{s}}$ is observed, indicating that the helical

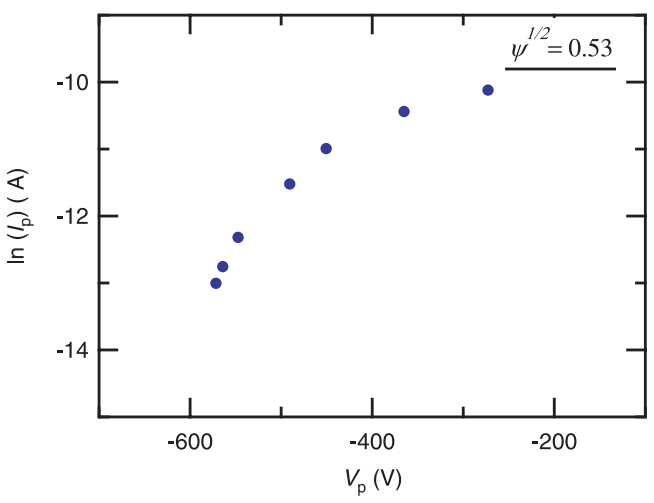

Fig. 2 Typical current-voltage $\left(I_{\mathrm{p}}-V\right)$ characteristics obtained with an electrostatic probe installed on the 11.5 cross section of Heliotron J. Measured curve clearly indicates a non-Maxwellian distribution of electron plasmas.

nonneutral plasma has not yet relaxed to its thermal equilibrium. Therefore, to obtain $n_{\mathrm{e}}$ values, we have used the simplest relationship between $I_{\mathrm{p}}$ and $n_{\mathrm{e}}$

$$
I_{\mathrm{p}} \sim e n_{\mathrm{e}} v_{\mathrm{e}} S
$$

where $v_{\mathrm{e}}$ is the electron speed, and $S$ is the probe area. Since the sum of $\varphi_{\mathrm{s}}$ and the kinetic energy of an electron is almost conserved, $v_{\mathrm{e}}$ can thus be approximately calculated from

$$
v_{\mathrm{e}}=\sqrt{\frac{2 e\left(\phi_{\mathrm{s}}-V_{\mathrm{acc}}\right)}{m_{\mathrm{e}}}},
$$

where $m_{\mathrm{e}}$ is the electron mass.

\subsection{Profiles of $\varphi_{\mathrm{s}}, I_{\mathrm{p}}$, and $\boldsymbol{n}_{\mathrm{e}}$}

Data were taken on the three different cross sections of Heliotron J. Figure 3 shows a typical set of measured profiles of $\varphi_{\mathrm{s}}, I_{\mathrm{p}}$, and $n_{\mathrm{e}}$. All plotted data are time-averaged values between $t=8$ and $9 \mathrm{~ms}$. Also, all coordinates for measurement points are translated into the normalized minor radius $\Psi^{1 / 2}$. Hence, $\Psi^{1 / 2}=0$ and 1 correspond to $R_{\mathrm{ax}}$ and the LCFS, respectively.

Substantial differences between the two values of $\varphi_{\mathrm{s}}$ measured in the upper and lower regions on each magnetic surface (at the same value of $\Psi^{1 / 2}$ ) are observed. Thus, $\varphi_{\mathrm{s}}$ is not constant on the HMS. The difference in $\varphi_{\mathrm{s}}$ is larger in the upper region of the HMS on the 6.5 poloidal cross section. This means that the equipotential surfaces have shifted upward from the HMS there. The absolute value of the magnitude of the difference in $\varphi_{\mathrm{s}}$, reaches about $500 \mathrm{~V}$ at the LCFS, whereas at $\Psi^{1 / 2} \sim 0.3$, the difference in $\varphi_{\mathrm{s}}$ almost disappears.

When examined carefully, all the measured profiles seem to have similar shapes. Also, $\phi_{\mathrm{s}} \sim V_{\text {acc }}$ near the helical magnetic axis. The measured $I_{\mathrm{p}}$, on the data on the 6.5 poloidal cross section is larger than the other values, possibly because the e-gun is located near that cross section. The value of $n_{\mathrm{e}}$ calculated from these data is of the order of 


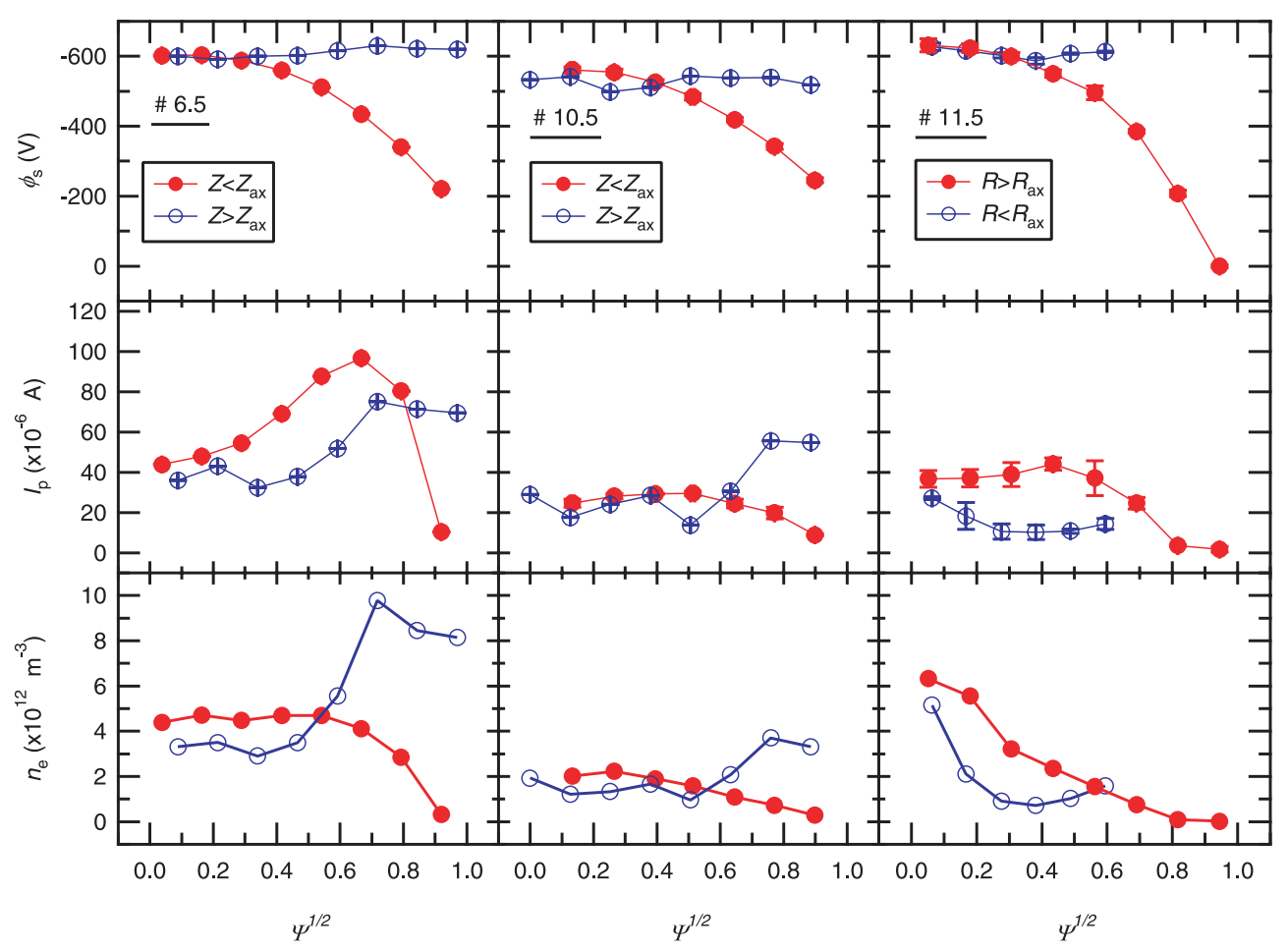

Fig. 3 Profiles of the space potential $\varphi_{\mathrm{s}}$ and probe current $I_{\mathrm{p}}$ of helical nonneutral plasmas confined on Heliotron J. Profiles of electron density $n_{\mathrm{e}}$ inferred from the measured $I_{\mathrm{p}}$ are also plotted.

$10^{12} \mathrm{~m}^{-3}$ on the HMS of Heliotron $\mathrm{J}$. This value is smaller than that of CHS experiments, although the magnitude of $B$ is the same, of the order of $0.1 \mathrm{kG}$.

Similar differences also appear in the measured $I_{\mathrm{p}}$, which can be clearly recognized in Fig. 3 . This reflects the profile of inferred $n_{\mathrm{e}}$, showing that $n_{\mathrm{e}}$ in the lower region is certainly larger than in the upper one. These results are similar to observations in past CHS experiments [7].

Next, we examine the variation in $n_{\mathrm{e}}$ on the HMS of Heliotron J. Since toroidally confined nonneutral plasmas can relax to the minimum energy state [12], more electrons should stay at the place on the HMS closest to the vacuum chamber wall. The measured data show that values of $n_{\mathrm{e}}$ in the lower region seem to be smaller in the core region where $\Psi^{1 / 2}<0.6$, whereas a completely opposite relationship is recognized in the outermost edge region where $\Psi^{1 / 2}>0.6$. However, such a crossover of $n_{\mathrm{e}}$ has not yet been justified. This is because line tracing of magnetic field lines shows that the toroidally averaged position of the magnetic axis of the HMS located almost on the center of the vacuum chamber in Heliotron J. Moreover, no apparent macroscopic shift of HMS in a particular direction occurs. The observed crossover of $n_{\mathrm{e}}$ is thus still an open questions and is examined in experiments that will be reported elsewhere.

\section{Two-dimensional Simulations}

To numerically examine the measured data in Fig. 3, we calculate $n_{\mathrm{e}}$ using a two-dimensional model. Since $\varphi_{\mathrm{s}}(r)$ has been obtained, $n_{\mathrm{e}}(r)$ can be approximately calculated from Poisson's equation: $\nabla^{2} \varphi_{\mathrm{s}}=-e n_{\mathrm{e}} / \epsilon_{0}$. Note that neither three-dimensional effects nor positive image charge induced on the inner surface of the vacuum chamber are considered. Figure 4 shows the resulting $n_{\mathrm{e}}(r)$. The calculated $n_{\mathrm{e}}(r)$ is of the same order as the measured value. Thus, within the experimental error, both $\varphi_{\mathrm{s}}$ and $n_{\mathrm{e}}$ are consistent with each other, which strongly suggests the existence of equilibrium in helical nonneutral plasmas having a helical magnetic axis.

However, some disagreements appear between the measurements and calculations. First, the peak position of the calculated $n_{\mathrm{e}}(r)$ is located at $z \sim-0.13$, but it is at $z \sim-0.1$ for the measured $n_{\mathrm{e}}(r)$. Second, apparent differences in the magnitude of $n_{\mathrm{e}}(r)$ are observed near the helical magnetic axis at $z \sim 0$. We consider the possible reasons. First, values of $n_{\mathrm{e}}$ near the helical magnetic axis may be overestimated. This is because the measured $I_{\mathrm{p}}$ is actually very small there, which can easily result in a considerable experimental error. Second, as mentioned above, we have solved Poisson's equation along only the radial direction (in fact, along the $x$-axis only) to avoid complicated calculations. If larger gradients of $\varphi_{\mathrm{s}}$ existed along both the $y$-axis and in the toroidal direction, such an overestimation of $n_{\mathrm{e}}$ would easily occur. In fact, larger variations of both $n_{\mathrm{e}}$ and $\varphi_{\mathrm{s}}$ have been clearly observed experimentally along the $x$-axis (that is, the direction of the minor radius $r$ ) near the LCFS (see also Figs. 3 and 4), whereas the variations become much smaller near the helical magnetic axis. Fur- 


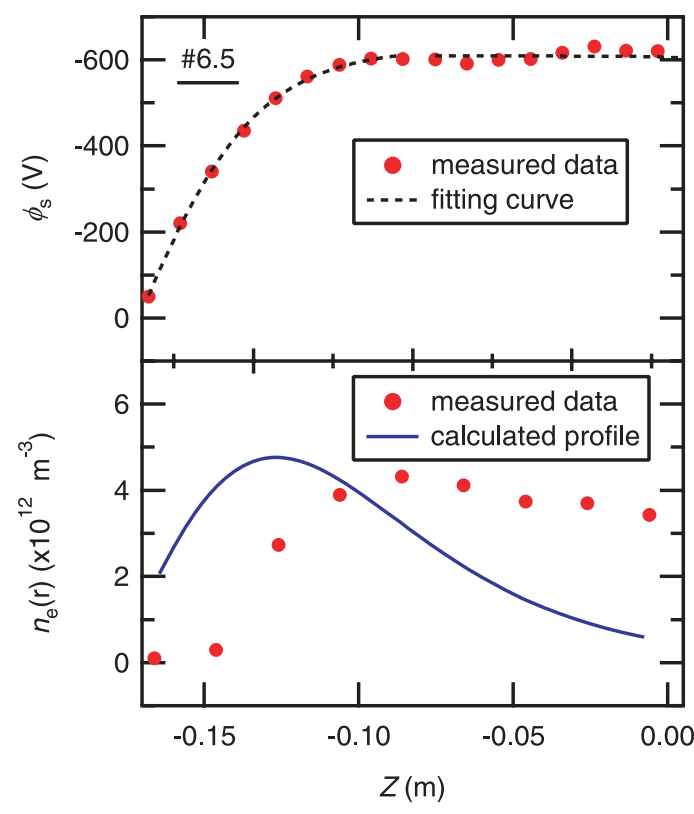

Fig. 4 Comparisons of calculated $n_{\mathrm{e}}$ (solid line) and fitting curve (dashed line) with measured data (solid circles) on the 6.5 cross section.

thermore, note that because Heliotron $\mathrm{J}$ has a helical magnetic axis, the spatial variation of $B$ along the toroidal direction would not be small. If $\varphi_{\mathrm{s}}$ also varied considerably along the toroidal direction at the plasma core, the calculated $n_{\mathrm{e}}$ would be larger. As a result, the discrepancy in Fig. 4 will be reduced. Further data along the helical magnetic axis is needed to be measured in order to answer this question.

\section{Summary}

For the first time, it is experimentally demonstrated that toroidal nonneutral plasmas can form on an HMS having a helical magnetic axis. The observed difference in $\varphi_{\mathrm{s}}$ becomes larger near the LCFS, which is similar to the results of past CHS experiments with helical nonneutral plasmas having plane magnetic axes. $\varphi_{\mathrm{s}}(r)$ and $n_{\mathrm{e}}(r)$ data are obtained on three different poloidal cross sections. Nonetheless, all of those data seem to have similar radial profiles. This suggests the existence of a helically symmetric equilibrium in helical nonneutral plasmas.

\section{Acknowledgment}

The authors are grateful to H. Hiramatsu and Y. Nishioka at KIT for preparing electrical circuits and to T. Senju at Kyoto University for his technical support. This work was performed under the auspices of the NIFS Research Collaboration, No. NIFS08KUHL018.

[1] F. M. Penning, Phisica 3, 873 (1936).

[2] H. Friedburg and W. Paul, Naturwissenschaft 38, 159 (1951).

[3] J. D. Daugherty, J. E. Eninger and G. S. Janes, Phys. Fluids 12, 2677 (1969).

[4] H. Himura, H. Wakabayashi, M. Fukao et al., Phys. Plasmas 11, 492 (2004).

[5] J. Kremer, T. S. Pedersen, Q. Marksteiner and R. Lefrancois, Phys. Rev. Lett. 97, 095003 (2006).

[6] H. Himura et al., P1-5 of this conference proceedings.

[7] H. Himura, H. Wakabayashi, Y. Yamamoto et al., Phys. Plasmas 14, 022507 (2007).

[8] M. Hahn, T. S. Pedersen, Q. Marksteiner and J. W. Berkery, Phys. Plasmas 15, 020701 (2008).

[9] R. G, Lefrancois and T. S. Pedersen, Phys. Plasmas 13, 120702 (2006).

[10] T. Obiki, T. Mizuuchi, K. Nagasaki et al., Nucl. Fusion 41, 833 (2001).

[11] H. Himura, H. Wakabayashi, Y. Yamamoto et al., Hyperfine Interact. 174, 83 (2007).

[12] A. H. Boozer, Phys. Plasmas 11, 4709 (2004). 